\title{
Recursive Least-Squares Estimation for the Joint Input-State Estimation of Linear Discrete Time Systems with Unknown Input
}

\author{
Talel. Bessaoudi ${ }^{1}$, Fayçal. Ben Hmida ${ }^{2}$ \\ Unité de recherche en Commande, Surveillance et Sûreté de fonctionnement des Systèmes (C3S) Tunis University- \\ ESSTT, 5 av. Taha Hussein BP 56-1008, Tunis, Tunisia \\ 1bessaouditalel@yahoo.fr, ${ }^{2}$ faycal.benhmida@esstt.rnu.tn
}

\begin{tabular}{|c|c|}
\hline Article Info & ABSTRACT \\
\hline Article history: & This paper presents a recursive least-squares approach to estimate \\
\hline Received Sep 9, 2014 & $\begin{array}{l}\text { simultaneously the state and the unknown input of linear time varying } \\
\text { discrete time systems with unknown input. The method is based on the }\end{array}$ \\
\hline Revised Jan 3, 2015 & assumption that no prior knowledge about the dynamical evolution of the \\
\hline Accepted Jan 15, 2015 & $\begin{array}{l}\text { input is available. The joint input and state estimation are obtained by } \\
\text { recursive least-squares formulation by applying the inversion lemmas. The }\end{array}$ \\
\hline Keyword: & $\begin{array}{l}\text { proposed filter is equivalent to recursive three step filter. To illustrate the } \\
\text { performance of the proposed filter an example is given. }\end{array}$ \\
\hline Information formulas & \\
\hline Inversion lemma & \\
\hline Least-squares & \\
\hline State estimation & \\
\hline Unknown input estimation & $\begin{array}{r}\text { Copyright (C) } 2015 \text { Institute of Advanced Engineering and Science. } \\
\text { All rights reserved. }\end{array}$ \\
\hline \multicolumn{2}{|l|}{ Corresponding Author: } \\
\hline \multicolumn{2}{|l|}{ Talel Bessaoudi, } \\
\hline \multicolumn{2}{|c|}{ Unité de recherche en Commande, Surveillance et Sûreté de fonctionnement des Systèmes (C3S) } \\
\hline \multicolumn{2}{|c|}{ Tunis University-ESSTT, 5 av. Taha Hussein BP 56-1008, Tunis, Tunisia } \\
\hline \multicolumn{2}{|c|}{ Email: bessaouditalel@yahoo.fr } \\
\hline
\end{tabular}

\section{INTRODUCTION}

During the last decades, the problem of unknown input filtering has received growing attention due to its applications in environmental state estimation [1], [2]. The unknown input filtering problem has treated in the literature by different approaches. The first approach assumes that the model for dynamical evolution of the unknown input is available. When the properties of the unknown input are known, the augmented state Kalman filter (ASKF) is a solution. To reduce computation costs of the ASKF, Friedland [2] proposed the two stage Kalman filter where the estimation of the state and unknown input are decoupled. The second approach treats the case when not have a prior knowledge about the dynamical evolution for the unknown input. Kitanidis [1] was the first to solve the problem using the linear unbiased minimum-variance. Darouach et al, [3] extend Kitanidis's filter using a paramaterizing technique to obtain an optimal filter (OEF). Hsieh [4] has developed an equivalent to Kitanidis's filter noted by robust-two stage Kalman filter (RTSKF). Later, Hsieh [5] developed an optimal minimum variance filter (OMVF) to solve the performance of degradation problem encountered in (OEF). Gillijns \& De Moor [6] has treated the problem to estimate the state in the presence of unknown input which affects only the systems model. They developed a recursive filter which is optimal in the sense of minimum-variance. This filter has been extended by the same authors [7] for joint input and state estimation to linear discrete-time systems with direct feedthrough where the state and the unknown input estimation are interconnected. This filter is called recursive three step filter (RTSF) and is limited to direct feedthrough matrix has full rank. Cheng et al, [8] proposed a recursive optimal filter with global optimality in the sense of unbiased minimum-variance over all unbiased estimators, but this filter is 
limited to estimate the state. Recently, the case of an arbitrary rank has been solved by Hsieh (2009) in the designed optimal filter [9], known as ERTSF (Extend RTSF). Other methods are proposed by Gillijns and Bart de Moor in [10], [11] and [12] which use least -squares (LS) technique and the information formulas.

In this paper, we present an unbiased minimum-variance estimation of the state and the unknown input. These estimates are obtained by solving the information formulas using the weighted least-squares method. The advantage of this method is to provide a direct estimate of the state and unknown input in a single block with a simple calculation.

The paper is organized as follow. Section 2, presents the problem under consideration and some preliminaries. In section 3 , we set up the design of the filter equation by recursively solving the weighted least-squares problem. An illustrative example is presented in section 4. Finally, in section 5 we conclude our obtained results.

\section{PROBLEM AND PRELEMINARIES}

\subsection{Problem Formulation}

Consider the linear stochastic discrete-time system with unknown input in the following form:

$$
\begin{aligned}
& x_{k+1}=A_{k} x_{k}+G_{k} d_{k}+w_{k} \\
& y_{k}=C_{k} x_{k}+H_{k} d_{k}+v_{k}
\end{aligned}
$$

where $x_{k} \hat{\mathrm{I}} \hat{\mathrm{A}}^{n}$ is the state vector, $d_{k} \hat{\mathrm{I}} \hat{\mathrm{A}}^{m}$ is the unknown input vector and $y_{k} \hat{\mathrm{I}} \hat{\mathrm{A}}^{p}$ is the measurement vector. The process noise $w_{k} \hat{\mathrm{I}} \hat{\mathrm{A}}^{n}$ and the measurement noise $v_{k} \hat{\mathrm{I}}^{p} \hat{\mathrm{A}}^{p}$ are assumed to be mutually uncorrelated zeros-mean white random signals with non singular covariance matrices $Q_{k}=e_{\varepsilon} v_{k} w_{k}^{T} \grave{H}_{k} 0$ and

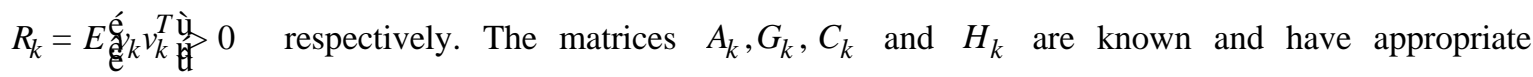
dimension. We assume that $\left(A_{k}, C_{k}\right)$ is observable, $p \geq m$ and the initial state is uncorrelated with the white noises processes $w_{k}$ and $v_{k}$. The initial state $x_{0}$ is a Gaussian random variable with $E\left[x_{0}\right]=\hat{x}_{0}$, $E\left[\left(x_{0}-\hat{x}_{0}\right)\left(x_{0}-\hat{x}_{0}\right)^{T}\right]=P_{0}$ where $E[$.$] denotes the expectation operator. Also, we assume that$ $\operatorname{rank}\left(C_{k} G_{k-1}\right)=\operatorname{rank}\left(G_{k-1}\right)$, the direct feedthrough matrix $H_{k}$ has an arbitrary rank .

The objective of this paper is to design an optimal recursive filter wich estimates both the system state $x_{k}$ and the unknown input $d_{k}$ based on the initial estimate $\hat{x}_{0}$ and the sequence of measurement $\left\{y_{0}, y_{1}, \ldots, y_{k}\right\}$. No prior knowledge about the dynamical evolution of $d_{k}$ is assumes to be available. Now we derive a Recursive Least Square (RLS) procedure that propagates a one step ahead predicted state estimate. For simplicity of derivations, we use a stochastic approach .We assume that an estimate $\hat{x}_{k / k-1}$ is available with covariance matrix $P_{k / k-1}=E\left[\left(x_{k}-\hat{x}_{k / k-1}\right)\left(x_{k}-\hat{x}_{k / k-1}\right)^{T}\right]$ and we seek for a weighted least square (WLS) that allows to estimate $\hat{x}_{k / k}$ based on $\hat{x}_{k / k-1}$ and the newly available measurement $y_{k}$.

The error estimation $X_{k / k-1}$ is given by:

$$
\chi_{R / k-1}:=x_{k}-\hat{x}_{k / k-1}
$$

Using (1), (2) and (3), we obtain the following equation:

$$
\left[\begin{array}{c}
\hat{x}_{k / k-1} \\
y_{k} \\
0
\end{array}\right]=\left[\begin{array}{ccr}
I_{n} & 0 & 0 \\
C_{k} & H_{k} & 0 \\
A_{k} & G_{k} & -I_{n}
\end{array}\right]\left[\begin{array}{c}
x_{k} \\
d_{k} \\
x_{k+1}
\end{array}\right]+\left[\begin{array}{c}
-\tilde{x}_{k / k-1} \\
v_{k} \\
w_{k}
\end{array}\right] .
$$


So, the corresponding WLS problem is given by

$$
\min _{x_{k}, d_{k}, x_{k+1}}\left\|\left[\begin{array}{c}
\hat{x}_{k / k-1} \\
y_{k} \\
0
\end{array}\right]-\left[\begin{array}{ccc}
I & 0 & 0 \\
C_{k} & H_{k} & 0 \\
A_{k} & G_{k} & -I_{n}
\end{array}\right]\left[\begin{array}{c}
x_{k} \\
d_{k} \\
x_{k+1}
\end{array}\right]\right\|_{W_{k}}^{2}
$$

where $\mathcal{W}_{k}$ denotes the weighting matrix.

From (5) the interpretation of an MVU (Unbiased Minimum-Variance) estimator is obtained by choosing $\mathcal{W}_{k}=\operatorname{diag}\left(P_{k / k-1}^{-1}, R_{k}^{-1}, Q_{k}^{-1}\right)$. The proposed solution of the LS problem (5) is given in the following form:

$$
\begin{aligned}
& \hat{d}_{k / k}=\mathcal{M}_{k}\left(y_{k}-C_{k} \hat{x}_{k / k-1}\right) \\
& \hat{x}_{k / k}=\hat{x}_{k / k-1}+K_{k}\left(y_{k}-C_{k} \hat{x}_{k / k-1}-H_{k} \hat{d}_{k / k}\right) \\
& \hat{x}_{k+1 / k}=A_{k} \hat{x}_{k / k}+G_{k} \hat{d}_{k / k}
\end{aligned}
$$

Where the gain matrices $\mathcal{M}_{k} \in \mathfrak{R}^{m \times p}$ and $K_{k} \in \mathfrak{R}^{n \times p}$ still have to be determined later.

\subsection{Preliminaries}

The following lemmas are essential for later developments.

\section{Lemma A.1 (The matrix inversion lemma [11]):}

Let $A \in \mathfrak{R}^{n \times n}, B \in \mathfrak{R}^{n \times m}, C \in \mathfrak{R}^{m \times n}$ and $D \in \mathfrak{R}^{m \times m}$ be real matrices. If $A, D-C A^{-1} B$ and $D$ are nonsingular, then $A-B D^{-1} C$ is non-singular, and $\left(A-B D^{-1} C\right)^{-1}=A^{-1}+A^{-1} B\left(D-C A^{-1} B\right)^{-1} C A^{-1}$.

The following formula provides a manner to invert a $2 \times 2$ block matrix based on the matrix inversion lemma, $\left[\begin{array}{cc}A & B \\ C & D\end{array}\right]^{-1}=\left[\begin{array}{cc}\left(A-B D^{-1} C\right)^{-1} & 0 \\ 0 & \left(D-C A^{-1} B\right)^{-1}\end{array}\right]\left[\begin{array}{ccc}I & -B D^{-1} \\ -C A^{-1} & I\end{array}\right]$.

Indeed, the diagonal entries of the first matrix on the right hand side of the equality sign can be computed using the matrix inversion lemma.

\section{Lemma A.2:}

Let $A \in \mathfrak{R}^{n \times n}, B \in \mathfrak{R}^{n \times m}$ and $C \in \mathfrak{R}^{m \times m}$ be real matrices. If $A, C$ are non-singular then, $A B\left(C+B^{T} A B\right)^{-1}=\left(A^{-1}+B C^{-1} B^{T}\right)^{-1} B C^{-1}$.

\section{FILTER DESIGN}

The calculation of the optimal matrices $\mathcal{M}_{k}$ and $K_{k}$ is addressed in the subsection 3.1 which call the measurement update, yields an estimate of $x_{k}$ and unknown input $d_{k}$. The time update of the state estimation is presented in subsection 3.2.

\subsection{Measurement Update}

The measurement update is derived from (5) by extracting the rows that depend only on $x_{k}$ and $d_{k}$. This yield,

$$
\min _{x_{k}, d_{k}}\left\|\left[\begin{array}{c}
\hat{x}_{k / k-1} \\
y_{k}
\end{array}\right]-\left[\begin{array}{cc}
I & 0 \\
C_{k} & H_{k}
\end{array}\right]\left[\begin{array}{l}
x_{k} \\
d_{k}
\end{array}\right]\right\|_{W_{1, k}}^{2}
$$


Where $\mathcal{W}_{1, k}=\operatorname{diag}\left(P_{k / k-1}^{-1}, R_{k}^{-1}\right)$ denotes the weighting matrix.

Now we derive an explicit update formula by solving the problem state and unknown input estimations. Firstly, note that (9) is equivalent to the least-squares problem

$$
\min _{X_{k}}\left\|Y_{k}-\mathcal{A}_{k} X_{k}\right\|_{W_{2, k}}^{2}
$$

Where

$$
\begin{aligned}
& \mathcal{A}_{k}=\left[\begin{array}{ll}
I & 0 \\
C_{k} & H_{k}
\end{array}\right], \Upsilon_{k}=\left[\begin{array}{c}
\hat{x}_{k / k-1} \\
y_{k}
\end{array}\right], \\
& X_{k}=\left[\begin{array}{l}
x_{k} \\
d_{k}
\end{array}\right] \text { and } \mathcal{W}_{2, k}=\operatorname{diag}\left(R_{k}^{-1}, P_{k / k-1}^{-1}\right)
\end{aligned}
$$

Using the Gauss-Markov theorem [13], the solution is written as:

$$
\hat{X}_{k}=\left(\mathcal{A}_{k}^{\mathcal{T}} \mathcal{W}_{2, k} \mathcal{A}_{k}\right)^{-1} \mathcal{A}_{\mathcal{K}}^{\mathcal{T}} \mathcal{W}_{2, k} \Upsilon_{k}
$$

Using (11) the covariance matrix $\left(\mathcal{A}_{\mathcal{K}}^{\mathcal{T}} \mathcal{W}_{2, \ell} \mathcal{A}_{k}\right)^{-1}$ follows as

$$
\left(\mathcal{A}_{k}^{\mathcal{T}} \mathcal{W}_{2, \ell} \mathcal{A}_{k}\right)^{-1}=\left[\begin{array}{ll}
P_{k / k-1}^{-1}+C_{k}^{T} R_{k}^{-1} C_{k} & C_{k}^{T} R_{k}^{-1} H_{k} \\
H_{k}^{T} R_{k}^{-1} C_{k} & H_{k}^{T} R_{k}^{-1} H_{k}
\end{array}\right]^{-1}
$$

In the next section we will determinate an unbiased estimate of the state and unknown input by seeking a solution to the equation (13).

\section{Lemma 3.1:}

The expression of the error covariance matrix $P_{k / k}^{d}$ is given by:

$$
P_{k / k}^{d}=\left(H_{k}^{T} \tilde{R}_{k}^{-1} H_{k}\right)^{-1}
$$

and the error covariance matrix of the state is given in the following form :

$$
P_{k / k}=P_{k / k-1}-K_{k}\left(\tilde{R}_{k}-H_{k} P_{k / k}^{d} H_{k}^{T}\right) K_{k}^{T}
$$

where

$$
\begin{aligned}
& \tilde{R}_{k}=C_{k} P_{k / k-1} C_{k}^{T}+R_{k} \\
& K_{k}=P_{k / k-1} C_{k}^{T} \tilde{R}_{k}^{-1}
\end{aligned}
$$

\section{Proof:}

Note that, $P_{k / k}$ and $P_{k / k}^{d}$ can be identified as error covariance matrices of $\hat{x}_{k / k}$ and $\hat{d}_{k / k}$, that is,

$$
\begin{aligned}
& P_{k / k}=E\left[\tilde{x}_{k / k} \tilde{x}_{k / k}^{T}\right], \\
& P_{k / k}^{d}=E\left[\tilde{d}_{k / k} \tilde{d}_{k / k}^{T}\right] .
\end{aligned}
$$


Where the inverse of $P_{k / k}$ and $P_{k / k}^{d}$ are given, respectively, by

$$
\begin{aligned}
P_{k / k}^{-1}=\left(P_{k / k-1}^{-1}+C_{k}^{T} R_{k}^{-1} C_{k}\right)-C_{k}^{T} R_{k}^{-1} H_{k}\left(H_{k} R_{k}^{-1} H_{k}\right)^{-1} \\
\times H_{k}^{T} R_{k}^{-1} C_{k} \\
\left(P_{k / k}^{d}\right)^{-1}=\left(H_{k}^{T} R_{k}^{-1} H_{k}\right)-H_{k}^{T} R_{k}^{-1} C_{k} \\
\times\left(P_{k / k-1}^{-1}+C_{k}^{T} R_{k}^{-1} C_{k}\right)^{-1} C_{k}^{T} R_{k}^{-1} H_{k}
\end{aligned}
$$

Then, by applying lemma A.1 the equation (13) is rewritten as follows:

$$
\begin{aligned}
& {\left[\begin{array}{cc}
P_{k / k-1}^{-1}+C_{k}^{T} R_{k}^{-1} C_{K} & C_{k}^{T} R_{k}^{-1} H_{k} \\
H_{k}^{T} R_{k}^{-1} C_{k} & H^{T} R_{k}^{-1} H_{k}
\end{array}\right]^{-1}=\left[\begin{array}{cc}
P_{k / k} & 0 \\
0 & P_{k / k}^{d}
\end{array}\right]} \\
& \times\left[\begin{array}{l}
I \\
-C_{k}^{T} R_{k}^{-1} H_{k}\left(H_{k}^{T} R_{k}^{-1} H_{k}\right)^{-1} \\
-H_{k}^{T} R_{k}^{-1} C_{k}\left(P_{k / k-1}^{-1}+C_{k}^{T} R_{k}^{-1} C_{K}\right)^{-1}
\end{array}\right]
\end{aligned}
$$

Applying the matrix inversion lemma A.1 to the information formulas (18) and (19), the error covariance matrix $P_{k / k}^{d}$ and $P_{k / k}$ are given in the following forms:

$$
\begin{aligned}
& P_{k / k}^{d}=\left[\begin{array}{c}
\left(H_{k}^{T} R_{k}^{-1} H_{k}\right)-H_{k}^{T} R_{k}^{-1} C_{k}\left(P_{k / k-1}^{-1}+C_{k}^{T} R_{k}^{-1} C_{k}\right)^{-1} \\
\times C_{k}^{T} R_{k}^{-1} H_{k}
\end{array}\right]^{-1} \\
& =\left(H_{k}^{T} R_{k}^{-1} H_{k}\right)^{-1}+\left(H_{k}^{T} R_{k}^{-1} H_{k}\right)^{-1} H_{k}^{T} R_{k}^{-1} C_{k} \times \\
& {\left[\left(P_{k / k-1}^{-1}+C_{k}^{T} R_{k}^{-1} C_{k}\right)-C_{k}^{T} R_{k}^{-1} H_{k}\left(H_{k}^{T} R_{k}^{-1} H_{k}\right)^{-1} H_{k}^{T} R_{k}^{-1} C_{k}\right]^{-1}} \\
& \times C_{k}^{T} R_{k}^{-1} C_{k}\left(H_{k}^{T} R_{k}^{-1} H_{k}\right)^{-1} \\
& =H_{k}^{-1}\left(R_{k}+C_{k} P_{k / k-1} C_{k}^{T}\right) H_{k}^{-T} \\
& =\left(H_{k}^{T} \tilde{R}_{k}^{-1} H_{k}\right)^{-1} \\
& P_{k / k}=P_{k / k-1}-K_{k}\left(\tilde{R}_{k}-H_{k} P_{k / k}^{d} H_{k}^{T}\right) K_{k}^{T} \\
& =\bar{P}_{k}+\bar{P}_{k} C_{k}^{T} R_{k}^{-1} H_{k} P_{k / k}^{d} H_{k}^{T} R_{k}^{-1} C_{k} \bar{P}_{k} \\
& \times\left(H_{k}^{T} R_{k}^{-1} H_{k}-H_{k}^{T} R_{k}^{-1} C_{k} \bar{P}_{k} C_{k}^{T} R_{k}^{-1} H_{k}\right)^{-1} H_{k}^{T} R_{k}^{-1} C_{k} \bar{P}_{k} \\
& P_{k / k}=\left[\begin{array}{c}
\left(P_{k / k-1}^{-1}+C_{k}^{T} R_{k}^{-1} C_{k}\right) \\
-C_{k}^{T} R_{k}^{-1} H_{k}\left(H_{k} R_{k}^{-1} H_{k}\right)^{-1} H_{k}^{T} R_{k}^{-1} C_{k}
\end{array}\right]^{-1} H_{k}
\end{aligned}
$$




$$
=P_{k / k-1}-K_{k} \tilde{R}_{k} K_{k}^{T}+K_{k} H_{k} P_{k / k}^{d} H_{k}^{T} K_{k}^{T}
$$

where

$$
\begin{aligned}
& \bar{P}_{k}=\left(P_{k / k-1}^{-1}+C_{k}^{T} R_{k}^{-1} C_{k}\right)^{-1} \\
& \bar{P}_{k} C_{k}^{T} R_{k}^{-1}=P_{k / k-1} C_{k}^{T} \tilde{R}_{k}^{-1} \\
& C_{k} P_{k / k-1}=\tilde{R}_{k} K_{k}^{T}
\end{aligned}
$$

The gain matrix $K_{k}$ that minimize the error covariance is given by

$$
K_{k}=P_{k / k-1} C_{k}^{T} \tilde{R}_{k}^{-1}
$$

Setting the Derivate of (25) with respect to $K_{k}$, we get

$$
\frac{\partial P_{k / k}}{\partial K_{k}}=\tilde{R}_{k} K_{k}^{T}+H_{k} P_{k / k}^{d} H_{k}^{T} K_{k}^{T}
$$

Let replace the $P_{k / k}^{d}$ by equation (14), we notice that $\frac{\partial P_{k / k}}{\partial K_{k}}=0$, therefore the gain $K_{k}$ minimize the trace of the matrix covariance $P_{k / k}$.

Lemma 3.2:

An unbiased estimate of the unknown input $d_{k}$ can be obtained in the following form:

$$
\hat{d}_{k / k}=\mathcal{M}_{k}\left(y_{k}-C_{k} \hat{x}_{k / k-1}\right)
$$

where

$$
\mathcal{M}_{k}=P_{k / k}^{d} H_{k}^{T} \tilde{R}_{k}^{-1}
$$

We consider the minimum-variance unbiased state estimation $\hat{x}_{k / k}$ given in the following form:

$$
\hat{x}_{k / k}=\hat{x}_{k / k-1}+K_{k}\left(y_{k}-C_{k} \hat{x}_{k-1}-H_{k} \hat{d}_{k / k}\right)
$$

\section{Proof:}

The equation (12) can be written as follows

$$
\begin{aligned}
{\left[\begin{array}{c}
\hat{x}_{k / k} \\
\hat{d}_{k / k}
\end{array}\right]=} & {\left[\begin{array}{cc}
P_{k / k-1}^{-1}+C_{k}^{T} R_{k}^{-1} C_{K} & C_{k}^{T} R_{k}^{-1} H_{k} \\
H_{k}^{T} R_{k}^{-1} C_{k} & H^{T} R_{k}^{-1} H_{k}
\end{array}\right]^{-1} } \\
& \times\left[\begin{array}{cc}
P_{k / k-1}^{-1} & C_{k}^{T} R_{k}^{-1} \\
0 & H_{k}^{T} R_{k}^{-1}
\end{array}\right]\left[\begin{array}{c}
\hat{x}_{k / k-1} \\
y_{k}
\end{array}\right] .
\end{aligned}
$$

Substituting (20) in (38), we obtain

$$
\hat{x}_{k / k}=P_{k / k} P_{k / k-1}^{-1} \hat{x}_{k / k-1}
$$


$+\left(P_{k / k} C_{k}^{T} R_{k}^{-1}-P_{k / k} C_{k}^{T} R_{k}^{-1} H_{k} \times\left(H_{k}^{T} R_{k}^{-1} H_{k}\right)^{-1} H_{k}^{T} R_{k}^{-1}\right) y_{k}$, and estimation of unknown input is given in the following form:

$$
\begin{gathered}
\hat{d}_{k / k}=P_{k / k}^{d} H_{k}^{T} R_{k}^{-1} y_{k}-P_{k / k}^{d} H_{k}^{T} R_{k}^{-1} C_{k}\left(P_{k / k-1}^{-1}+C_{k}^{T} R_{k}^{-1} C_{k}\right)^{-1} \\
\times\left(P_{/ k-1}^{-1} x_{k / k-1}+C_{k}^{T} R_{k}^{-1} y_{k}\right) .
\end{gathered}
$$

Let us apply the lemma A.1 and A.3 to the equation (39) and (40), the estimate of the unknown input is given by:

$$
\begin{aligned}
& \hat{d}_{k / k}=P_{k / k}^{d} H_{k}^{T} R_{k}^{-1} y_{k}-P_{k / k}^{d} H_{k}^{T} R_{k}^{-1} C_{k} \\
& \times\left(P_{k / k-1}^{-1}+C_{k}^{T} R_{k}^{-1} C_{k}\right)^{-1}\left(P_{/ k-1}^{-1} \hat{x}_{k / k-1}+C_{k}^{T} R_{k}^{-1} y_{k}\right) .
\end{aligned}
$$

where

$$
\begin{aligned}
& P_{k / k}^{d} H_{k}^{T} R_{k}^{-1} C_{k}\left(P_{k / k-1}^{-1}+C_{k}^{T} R_{k}^{-1} C_{k}\right)^{-1} P_{k / k-1}^{-1} \hat{x}_{k / k-1} \\
& =\left(H_{k}^{T} \tilde{R}_{k}^{-1} H_{k}\right) H_{k}^{T} \tilde{R}_{k}^{-1} C_{k} \hat{x}_{k / k-1} . \\
& P_{k / k}^{d} H_{k}^{T}\left[R_{k}^{-1}-R_{k}^{-1} C_{k}\left(P_{k / k-1}^{-1}+C_{k}^{T} R_{k}^{-1} C_{k}\right)^{-1} C_{k}^{T} R_{k}^{-1}\right] y_{k} \\
& =P_{k / k}^{d} H_{k}^{T}\left(R_{k}+C_{k} P_{k / k-1} C_{k}^{T}\right)^{-1} y_{k} . \\
& \hat{d}_{k / k}=\left(H_{k}^{T} \tilde{R}_{k}^{-1} H_{k}\right) H_{k}^{T} \tilde{R}_{k}^{-1}\left(y_{k}-C_{k} \hat{x}_{k / k-1}\right)
\end{aligned}
$$

Remark 3.1: to evaluate the performance of the filter in case where $H_{k}$ has an arbitrary rank we use the heuristic extension presented in [9] by replacing equation (14) and (36) by:

$$
\mathcal{M}_{k}=P_{k / k}^{d} H_{k}^{T} \tilde{R}_{k}^{-1}, \quad P_{k / k}^{d}=\left(H_{k}^{T} \tilde{R}_{k}^{-1} H_{k}\right) \dagger
$$

where $^{\dagger}$ the Moor-Penrose is generalized inverse

$$
M^{\dagger}=\left(M^{T} M\right)^{-1} M^{T}
$$

The state estimation $\hat{x}_{k / k}$ is given in the following form:

$$
\begin{aligned}
& \hat{x}_{k / k}=P_{k / k} P_{k / k-1}^{-1} \hat{x}_{k / k-1}+ \\
& \left(P_{k / k} C_{k}^{T} R_{k}^{-1}-P_{k / k} C_{k}^{T} R_{k}^{-1} H_{k}\left(H_{k}^{T} R_{k}^{-1} H_{k}\right)^{-1} H_{k}^{T} R_{k}^{-1}\right) y_{k}
\end{aligned}
$$

Using the inversion lemma A.1 and A.3 we can show that:

$$
\begin{aligned}
& P_{k / k} P_{k / k-1}^{-1} \hat{x}_{k / k-1}=\hat{x}_{k / k-1}-P_{k / k-1} C_{k}^{T} \tilde{R}_{k}^{-1} C_{k} \hat{x}_{k / k-1} \\
& +P_{k / k-1} C_{k}^{T} \tilde{R}_{k}^{-1} H_{k}\left(H_{k}^{T} \tilde{R}_{k}^{-1} H_{k}\right)^{-1} H_{k}^{T} \tilde{R}_{k}^{-1} C_{k} \hat{x}_{k / k-1} . \\
& P_{k / k} C_{k}^{T}\left(R_{k}^{-1}-R_{k}^{-1} H_{k}\left(H_{k}^{T} R_{k}^{-1} H_{k}\right)^{-1} H_{k}^{T} R_{k}^{-1}\right) y_{k} \\
& =P_{k / k-1} C_{k}^{T} \tilde{R}_{k}^{-1} y_{k}-P_{k / k-1} C_{k}^{T} \tilde{R}_{k}^{-1} H_{k}\left(H_{k}^{T} \tilde{R}_{k}^{-1} H_{k}\right)^{-1} \\
& \quad \times H_{k}^{T} \tilde{R}_{k}^{-1} y_{k} .
\end{aligned}
$$




$$
\begin{aligned}
& \hat{x}_{k / k}=\hat{x}_{k / k-1}-P_{k / k-1} C_{k}^{T} \tilde{R}_{k}^{-1} C_{k} \hat{x}_{k / k-1} \\
& +P_{k / k-1} C_{k}^{T} \tilde{R}_{k}^{-1} H_{k}\left(H_{k}^{T} \tilde{R}_{k}^{-1} H_{k}\right)^{-1} H_{k}^{T} \tilde{R}_{k}^{-1} C_{k} \hat{x}_{k / k-1} \\
& +P_{k / k-1} C_{k}^{T} \tilde{R}_{k}^{-1} y_{k}-P_{k / k-1} C_{k}^{T} \tilde{R}_{k}^{-1} H_{k}\left(H_{k}^{T} \tilde{R}_{k}^{-1} H_{k}\right)^{-1} \\
& \quad \times H_{k}^{T} \tilde{R}_{k}^{-1} y_{k} \cdot \\
& \hat{x}_{k / k}=\left(I-P_{k / k-1} C_{k}^{T} \tilde{R}_{k}^{-1} C_{k}\right) \hat{x}_{k / k-1}+P_{k / k-1} C_{k}^{T} \tilde{R}_{k}^{-1} y_{k} \\
& -P_{k / k-1} C_{k}^{T} \tilde{R}_{k}^{-1} H_{k}\left(H_{k}^{T} \tilde{R}_{k}^{-1} H_{k}\right)^{-1} H_{k}^{T} \tilde{R}_{k}^{-1}\left(y_{k}-C_{k} \hat{x}_{k / k-1}\right)
\end{aligned}
$$

\subsection{Time Update}

Firstly, we extract from (4) the equation that depends on $x_{k+1}$.

$$
x_{k+1}=A_{k} x_{k}+G_{k} d_{k}+w_{k}
$$

Second substituting $x_{k}$ and $d_{k}$ for their LS estimates $\hat{x}_{k / k}$ and $\hat{d}_{k / k}$ obtained during the measurement update (41) and (50). Then, we obtain

$$
A_{k} \hat{x}_{k / k}+G_{k} \hat{d}_{k / k}=x_{k+1}-\left(A_{k} x_{k / k}+G d_{k / k}^{g}+w_{k}\right)
$$

The corresponding LS problem is given by

$$
\min \left\|x_{k+1}-A_{k} \hat{x}_{k / k}-G_{k} \hat{d}_{k}\right\|_{W_{3, k}}
$$

Where $\mathcal{W}_{3, k}$ denotes the weighting matrix which we choose

$$
W_{3, k}=\left(E\left[\left(A_{k} \tilde{x}_{k / k}+G_{k} \tilde{d}_{k / k}+w_{k}\right)\left(A_{k} \tilde{x}_{k / k}+G_{k} \tilde{d}_{k / k}+w_{k}\right)^{T}\right]\right)^{-1}
$$

From equation (53)

$$
\hat{x}_{k+1 / k}=A_{k} \hat{x}_{k / k}+G_{k} \hat{d}_{k / k}
$$

The error estimation $\tilde{x}_{k+1 / k}$ is given by

$$
\begin{aligned}
& \tilde{x}_{k+1 / k}=x_{k+1}-\hat{x}_{k+1 / k} \\
& =A_{k} \tilde{x}_{k / k}+G_{k} \tilde{d}_{k / k}+w_{k}
\end{aligned}
$$

In consequence, the covariance matrix of $\hat{x}_{k+1 / k}$ is given by:

$$
\begin{aligned}
& P_{k+1 / k}=E\left[\begin{array}{ll}
\tilde{x}_{k+1 / k} & x_{k+1 / k}^{T}
\end{array}\right] \\
& =\left[\begin{array}{ll}
A_{k} & G_{k}
\end{array}\right]\left[\begin{array}{cc}
P_{k / k} & P_{k / k}^{x d} \\
P_{k / k}^{d x} & P_{k / k}^{d}
\end{array}\right]\left[\begin{array}{c}
A_{k}^{T} \\
G_{k}^{T}
\end{array}\right]+Q_{k}
\end{aligned}
$$

It follows from (35) that $\tilde{d}_{k / k}$ is given by: 


$$
\begin{aligned}
& \tilde{d}_{k / k}=\left(I-M_{k} H_{k}\right) d_{k}-M_{k}\left(C_{k} \tilde{x}_{k / k-1}+v_{k}\right) \\
& P_{k / k}^{x d}=\left(P_{k / k}^{x d}\right)^{T}=E\left[\tilde{x}_{k / k} \tilde{d}_{k / k}\right] .
\end{aligned}
$$

Using (56) and (60), it follows that

$$
P_{k / k}^{X d}=-K_{k} H_{k} P_{k / k}^{d}
$$

\section{ILLUSTRATIVE EXAMPLE}

To show the proposed results, the numerical example given by Darouach, Zasadzinki and Boutayeb (2003) is considered, where the parameters of systems (1) and (2) are given as follows, the parameters of the system (1) and (2) are given by:

$$
\begin{aligned}
A_{k} & =\left[\begin{array}{cc}
-0.0005 & -0.0084 \\
0.0517 & 0.8069
\end{array}\right], C_{k}=\left[\begin{array}{cc}
1 & 0 \\
0 & 1
\end{array}\right], \\
G_{k} & =\left[\begin{array}{ll}
0.0129 & 0 \\
-1.2504 & 0
\end{array}\right], Q_{k}=\left[\begin{array}{ll}
0.0036 & 0.0342 \\
0.0342 & 0.3249
\end{array}\right] R_{k}=\left[\begin{array}{cc}
0.01 & 0 \\
0 & 0.16
\end{array}\right] .
\end{aligned}
$$

Without loss of generality, the initial state and its estimate are both assumed to be zero, and the initial covariance is given by $P_{0}=\operatorname{diag}(10,200)$. The unknown input are given by

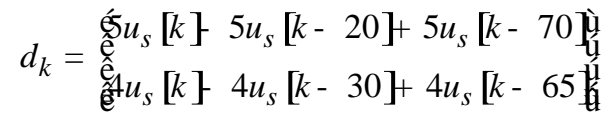

where $u_{s}[k]$ is the unit-step function. In this example, we assume that the simulation time is 100 time step.

$$
H_{k}^{1}=\left[\begin{array}{ll}
1 & 0 \\
0 & 0
\end{array}\right], H_{k}^{2}=\left[\begin{array}{ll}
1 & 0 \\
1 & 0
\end{array}\right] \text { and } H_{k}^{3}=\left[\begin{array}{ll}
1 & 0 \\
0 & 1
\end{array}\right]
$$

Case 1: $H_{k}=H_{k}^{1}$
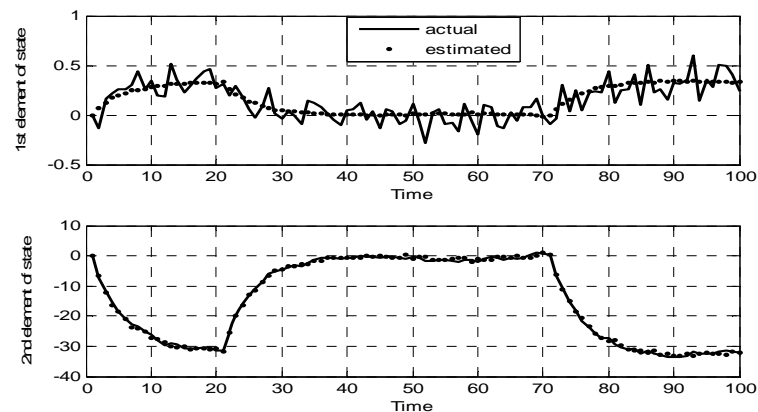

Figure 1. Actual and estimated value of the state 

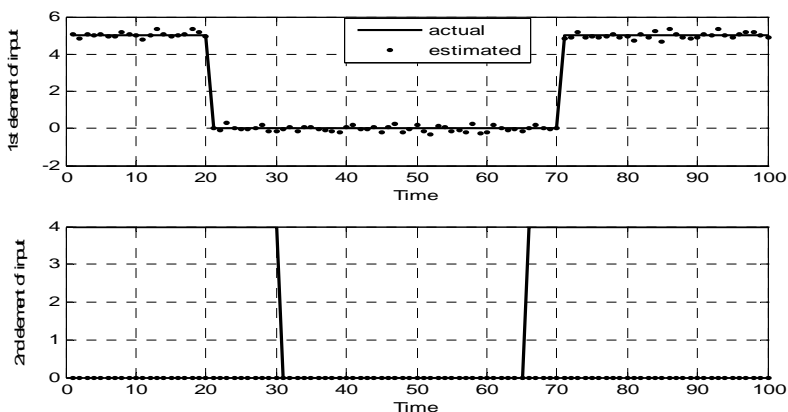

Figure 2. Actual and estimated value of the input

Case 2: $H_{k}=H_{k}^{2}$
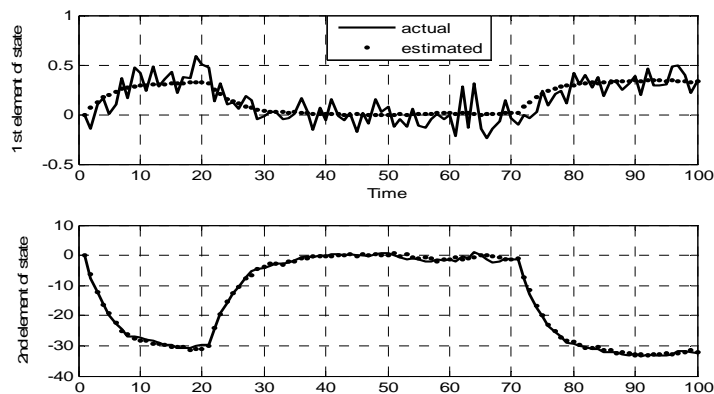

Figure 3. Actual and estimated value of the state
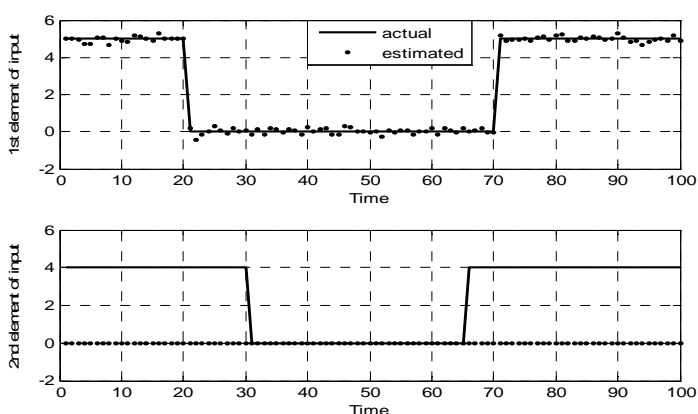

Figure 4. Actual and estimated value of the input 
Case 3: $H_{k}=H_{k}^{3}$
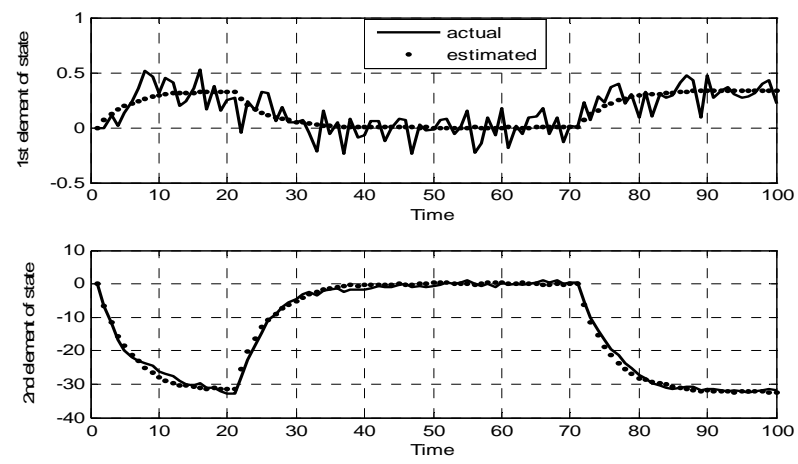

Figure 5. Actual and estimated value of the state
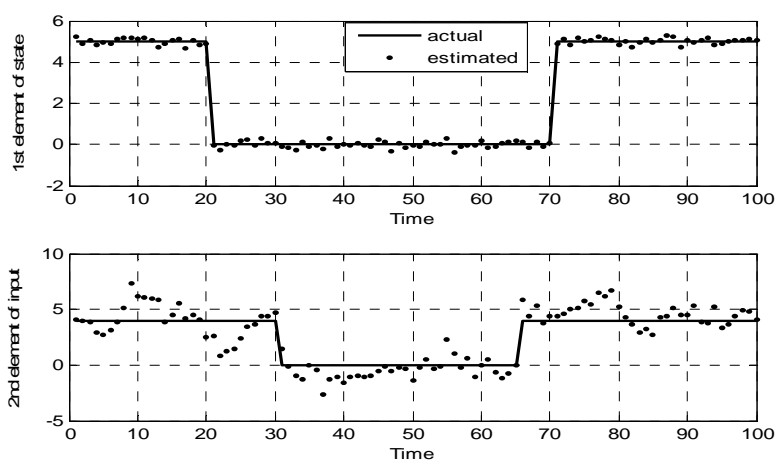

Figure 6. Actual and estimated value of the input

Table 1. Performance of the proposed filter

\begin{tabular}{ccccccc}
\hline$H_{k}$ & \multicolumn{4}{c}{$R M S E$} & \multicolumn{3}{c}{ Trace P } \\
& $\tilde{x}_{k}^{1}$ & $\tilde{x}_{k}^{2}$ & $\tilde{d}_{k}^{1}$ & $\tilde{d}_{k}^{2}$ & $\operatorname{tracP}_{k}^{X}$ & $\operatorname{tracP}{ }_{k}^{d}$ \\
\hline$H_{k}^{1}$ & 0.031 & 0.161 & 0.024 & - & 0.430 & 0.0135 \\
$H_{k}^{2}$ & 0.083 & 0.064 & 0.120 & 4.000 & 0.459 & 0.0136 \\
$H_{k}^{3}$ & 0.071 & 0.014 & 0.018 & 0.123 & 1.014 & 1.1847 \\
\hline
\end{tabular}

In table 1 , the root square errors (RMSE) of the state $x_{k}=\left[\begin{array}{ll}x_{k}^{1} & x_{k}^{2}\end{array}\right]^{T}$ and the inputs $d_{k}=\left[\begin{array}{ll}d_{k}^{1} & d_{k}^{2}\end{array}\right]^{T}$ are given as well as the traces of their steady-input and state estimation error covariance. For example the RMSE of the first component of sate vector is calculated by

$$
\operatorname{RMSE}\left(\tilde{x}_{1, k}\right)=\sqrt{\frac{1}{N} \sum_{k=1}^{N}\left(x_{1, k}-\hat{x}_{1, k}\right)^{2}}
$$

In figures 1, 3 and 5 we plot that actual and the estimated value of the two element of the state vector $x_{k}=\left[\begin{array}{ll}x_{k}^{1} & x_{k}^{2}\end{array}\right]^{T}$ in the three cases. 
In figures 2, 4 and 6 we plot that actual and the estimated value of the two elements of the first and second element of the input vector $d_{k}=\left[\begin{array}{ll}d_{k}^{1} & d_{k}^{2}\end{array}\right]^{T}$ in the three cases.

According to Table1, Figures 1, 2, 3, 4, 5 and 6 we may conclude the following results:

If the direct feedthrough matrix has full rank $H_{k}=H_{k}{ }^{3}$ then the proposed filter (RLSF) guarantees an estimate with a small value (RMSE) for the two components of the two vectors of state and unknown input.

\section{CONCLUSION}

In this paper, the recursive filter design for systems with unknown input via the least-squares technique is proposed. The obtained recursive filter is named RLSF. This solution is based on the technique least square when the direct feedthrough matrix of the unknown input has an arbitrary rank. An application of the proposed filter has been shown by an illustrative example. This filter may be used in resolving Fault Detection and Isolation.

\section{REFERENCES}

[1] P.K. Kitanidis. "Unbaised minimum variance linear state estimation”. Automatica. vol. 23, no. 6, pp. 775-778, 1987.

[2] B. Friedland. "Treatment of bias in recursive filtering". IEEE Transactions Control. vol. 14, pp. 359- 367, 1969.

[3] M. Darouach, M. Zasadzinki, and M. Boutayeb. "Extension of minimum variance estimation for systems with unknown inputs”. Automatica. vol. 39, no. 5 pp. 867-876, 2003.

[4] C.S. Hsieh. "Robust two-stage Kalman filters for systems with unknown inputs". IEEE Transaction on Automatica Control. vol. 45, no. 12, pp. 2374-23778, 2000.

[5] C.S. Hsieh. "Optimal minimum-variance filtering for systems with unknown inputs". In proccedings of the $6^{\text {th }}$ World Congress on Intelligent Control and Automatica (WCICA'06). Vol. 1 Dalian, Ghina, pp. 1870-1874, 2006.

[6] S. Gillijns and B. Moor. "Unbaised minimum-variance input and state estimation for linear discret-time systems with direct feedthrough”. Automatica. vol. 43, no. 5, pp. 934-937, 2007.

[7] S. Gillijns and B. Moor. "Unbaised minimum-variance input and state estimation for linear discret-time systems". Automatica. vol. 43, no. 1, pp. 111-116, 2007.

[8] Y. Cheng, H. Ye, Y. Wang, and D. Zhou. "Unbaised minimum-variance state estimation for linear discret-time systems with unknown input”. Automatica. Vol. 45, no. 2, pp 485-491, 2009.

[9] C.S. Hsieh. "Extension of unbiased minimum-variance input and state estimation for systems with unknown input". Automatica. vol. 45, no pp. 2149-2153, 2009.

[10] S. Gillijns and B. Moor. "Recursive Least-Squares Estimation for Systems with Unknown Inputs". Internel report. Accepted for publication in IEEE Transaction on Automatic control. 2007.

[11] S. Gillijns. "Kalman Filtering techniques for systems inversion and data assimilation". Katholieke universiteit leuven. 2007.

[12] S. Gillijns. "Information, Covariance and Square-Root filtering in the Presence of Unknown input”. Internal report. www.esat.kuleuven.be/scd. 2006.

[13] T. Kailath, A.H. Sayed, and B. Hassibi. "Linear Estimation”, Prentice -Hall, Englewood Cliffs, NJ, USA. 2000.

[14] Harish J. Palanthandalam-Madapusiand and Dennis S. Bernstein. "Unbaised Minimum-Variance Filtering for Input Reconstruction”. American Control Conference. New York City, USA. 2007. 